\title{
Multispeckle diffusing-wave spectroscopy: a tool to study slow relaxation and time-dependent dynamics
}

\author{
Virgile Viasnoff and François Lequeux \\ L.P.M UMR7615 CNRS ESPCI 10 rue Vauquelin 75231 Paris, France \\ D. J. Pine \\ Departments of Chemical Engineering and Materials, \\ University of California, Santa Barbara, CA 93106-5080
}

(Dated: October 25, 2018)

\begin{abstract}
A multispeckle technique for efficiently measuring correctly ensemble-averaged intensity autocorrelation functions of scattered light from non-ergodic and/or non-stationary systems is described. The method employs a CCD camera as a multispeckle light detector and a computer-based correlator, and permits the simultaneous calculation of up to 500 correlation functions, where each correlation function is started at a different time. The correlation functions are calculated in real time and are referenced to a unique starting time. The multispeckle nature of the CCD camera detector means that a true ensemble average is calculated; no time averaging is necessary. The technique thus provides a "snapshot" of the dynamics, making it particularly useful for non-stationary systems where the dynamics are changing with time. Delay times spanning the range from $1 \mathrm{~ms}$ to $1000 \mathrm{~s}$ are readily achieved with this method. The technique is demonstrated in the multiple scattering limit where diffusing-wave spectroscopy theory applies. The technique can also be combined with a recently-developed two-cell technique that can measure faster decay times. The combined technique can measure delay times from $10 \mathrm{~ns}$ to $1000 \mathrm{~s}$. The method is peculiarly well suited for studying aging processes in soft glassy materials, which exhibit both short and long relaxation times, non-ergodic dynamics, and slowly-evolving transient behavior.
\end{abstract}

PACS numbers: 07.60.-j, 78.35.+c, 81.40.Cd

\section{INTRODUCTION}

Dynamic light scattering has proven to be a powerful tool for investigating the dynamics of colloidal suspensions, emulsions, foams, and other complex fluids. Over the past decade, dynamic light scattering (DLS) has been increasingly applied to glassy systems with slow, and often non-ergodic, dynamics. Slow dynamics pose experimental difficulties for DLS, particularly with respect to the stability of the lasers, and sometimes for the light detection system. Nonergodic samples pose even more serious experimental difficulties, which are exacerbated by the presence of slow dynamics. One way of addressing the problem of long data acquisition times is to use an array detector. More importantly, an array detector can also address the problem of non-ergodic samples if the optics are designed so that the different elements in the array detector constitute essentially statistically independent samplings of the system under study.

In this article, we describe a method which employs a charge-coupled device (CCD) array detector for performing dynamic light scattering on systems that exhibit strong multiple light scattering. While the basic method we describe is applicable to single as well as multiple light scattering, our focus here is on multiple dynamic light scattering, also known as diffusing-wave spectroscopy (DWS) [1, 2, 3], where the methods we describe are most effective.

\section{BACKGROUND}

In conventional DWS (or DLS), the basic idea is to illuminate a sample with a coherent beam of light from a laser and to measure the temporal fluctuations in the resulting speckle pattern of scattered light. In a typical experiment, the optics for collecting the scattered light is arranged in such a way that the intensity of a single speckle is measured. By focusing on a single speckle, the amplitude of the fluctuating intensity is optimized to obtain a strongly fluctuating signal. The temporal duration of a typical fluctuation, i.e. the lifetime of a speckle, is determined by the time it takes a particle to move such that the phase of the scattered light is changed by approximately unity (or $\pi$ ). The dynamics of the scattered light, and hence the dynamics of the scatterers, can be more quantitatively characterized by the temporal autocorrelation function of the scattered intensity. In many cases, the autocorrelation function can be analyzed so that the time dependent mean square displacement of the scatterers is extracted [3].

There are two important keys to the success of the conventional analysis that we wish to focus on here. First, the process must be stationary, at least for the duration of the experiment. That is, the dynamics of the system cannot change significantly during this time period. For example, the gelation process of a colloidal suspension can be monitored provided that the particle dynamics slow down (due to the increase of the elastic modulus) occurs on time scales longer than the measurement period. 
In general an accurate measurement of the correlation function requires that the fluctuations in the intensity be measured over many fluctuation periods (e.g. $\sim 10^{4}$ speckle lifetimes to achieve $\sim 1 \%$ accuracy). Thus, in this case, the particle dynamics must not change significantly over a time period of $\sim 10^{4}$ speckle lifetimes.

Second, the system must be ergodic. For a light scattering experiment, a system is ergodic if each speckle samples the same distribution of light intensities as the entire ensemble of speckles over the period of time that data is acquired (i.e. over the duration of the experiment). While this condition is satisfied for a large number of systems, there are certain systems with very slow glassy dynamics that are of interest but which, nevertheless, are not ergodic in the sense described above. Such systems pose a problem for light scattering and special steps must be taken in order to assure that meaningful correlation functions, which represent dynamics characteristic of the entire system, are measured [4, [5, 6].

The problem for light scattering presented by nonergodic samples was first understood in a clear fashion by Pusey and van Megen who, at the same time, presented a clever method of obtaining correctly averaged temporal autocorrelation functions 溲. This technique has proven to be very useful but has some drawbacks: it sacrifices some signal-to-noise in order to obtain a useful distribution of light intensities, and the dynamics of the system must not change significantly over the duration of the experiment [4, 6, 6. Moreover, it suffers from the same limitation as normal DLS experiments in that data must be collected over a time scale that is much longer that the longest time scale of the fluctuations one is trying to measure.

Other schemes have been developed which overcome some of these difficulties, but not without a significant cost. The sample can be translated or rotated, for example, so that an ensemble average is obtained by brute force [6]. However, the rotation or translation introduces an artificial characteristic time scale into the correlation function, namely the time scale over which the system samples new speckles by virtue of the motion. Care must be taken to assure that this time scale is much longer than any dynamical time scale of the system one is interested in measuring. And once again, the duration of the experiment must be such that averaging occurs over a time scale that is long compared to the time scale introduced by the sample motion (this amounts to assuring that a sufficiently large number of speckles are sampled - e.g. $\sim 10^{4}$ - to obtain the desired level of accuracy - e.g. $\sim 1 \%$ ). Another method, developed recently by Scheffold et al. [7, 8, 9], uses two cells, one containing the sample with the slow nonergodic dynamics that is being studied, and another containing diffusing particles with ergodic dynamics, which serves to spatially average the nonergodic signal. This technique is only useful for experiments involving strong multiple scattering, but nevertheless provides a simple and efficient means of dealing with nonergodic systems. However, the only time scales probed in the primary cell are those that are shorter than the diffusive time scale set by the secondary cell.

An attractive alternative to the techniques described above for dealing with nonergodic samples is to use a CCD array, conveniently contained in a commercial CCD camera, as a light detector 110, 11, 12, 13, 14, 15. This allows the intensity from a large number of speckles to be simultaneously and independently measured. A time series of images of scattered light obtained from the CCD camera are stored in a computer and processed such that each pixel in the CCD array serves as an input to its own correlator. Since the fluctuation of each speckle is independent of the other speckles, processing scattered intensity data collected with a CCD camera is like have $N$ independent samplings of the data, where $N$ is the number of speckles in the field of the CCD array. If $N$ is sufficiently large, e.g. $N \sim 10^{4}$, then a complete ensemble average is obtained without any need to sample the system over times any longer than the actual dynamical time scales that are under study. Thus, a direct ensemble average can be obtained without any need for time-averaging whatsoever. This approach solves several problems at once. First, it solves the nonergodicity problem because a full ensemble average (of $\sim 10^{4}$ samples) is obtained. Second, the process under study need not be a stationary process because no time averaging is necessary, provided a sufficiently large number of speckles are used. Thus, an essentially instantaneous snapshot of the system dynamics can be taken. If those dynamics change in time, they can be followed simply by measuring temporal autocorrelation functions at different starting times.

There are two practical limitations of the CCDcamera-based technique. First, the minimum delay time for which the correlation function can be measured is set by the frame rate of the camera. In the experiments described in this article, we use a frame rate of 500 frames per second. Thus, delay times as small as a few milliseconds are accessible. It is interesting and important to note that real time processing of the images is necessary since, to our knowledge, no commercial buffer memory is able to store them for the entire sequence of frames obtained in the course of a single experiment, which is typically on the order of $10^{5}$ to $10^{7}$ frames. Recent advances in microprocessor speed allows real time computation of the correlation function. Hence the limiting factor for frame rate is the PCI bus velocity for transferring data from the acquisition board to the computer RAM.

The second limitation of the technique is the limited sensitivity of the CCD array used, as compared to that of a photomultiplier. This means that relatively strong scattered light signals are needed. In practice a power of $400 \mathrm{~mW}$ gives a satisfactory signal to noise ratio for a frame rate of 500 per seconds.

In this article we first describe the CCD optical set up and calculational scheme for our computer-based correlator. In the next section we explain how a single camera can be used to take up to 500 correlation functions simultaneously, with the reference starting time shifted by 
different amounts. We then show how the CCD camera technique can be coupled with the double cell technique to extend the time window over which measurements can be made from $10^{-8} \mathrm{~s}$ to $10^{4} \mathrm{~s}$.

\section{EXPERIMENTAL SETUP}

The sample is illuminated using polarized light from an argon-ion laser operating at a wavelength of $\lambda=514 \mathrm{~nm}$. Its maximal output power is 1.6 Watts. The laser beam is expanded to a diameter of approximately $1 \mathrm{~cm}$ and is incident on the sample cell, as shown in Fig. 1. Multiply scattered light is collected by a $50-\mathrm{mm}$ Nikon camera lens and focused onto a iris diaphragm. The lens is set up so that a one-to-one image of the scattered light from the output plane of the sample cell is formed on the diaphragm. The imaging of the output plane of the sample onto the pinhole provides a convenient means for collecting data from different parts of the sample. This feature is particularly useful in samples like gels or very viscous solutions where defects or small particles of dust may hinder the measurement; this set-up allows one to avoid defected regions of the sample. A polarizing cube is placed between the lens and the diaphragm to protect the camera from stray light.

The low sensitivity of CCD arrays relative to photomultiplier tubes means that the sample must be strongly illuminated. However, the incident light intensity must not be so high as to heat the sample. Therefore, it is crucial to collect as much scattered light as possible. We use a camera lens of $50 \mathrm{~mm}$ with a numerical aperture of 1.7 .

The scattered light is detected by a Dalsa CCD camera, model CAD1-128A, which is placed approximately $d \simeq 15 \mathrm{~cm}$ behind the diaphragm. It is an 8-bit camera which we run at 500 frames per second. The images are transferred to a computer running at $500 \mathrm{MHz}$ using a National Instruments data acquisition board, model PCI1422. The combined speeds of the computer and data acquisition boards are sufficiently high to allow data to be analyzed in real time.

Data analysis includes calculation of the ensembleaveraged temporal correlation function of the scattering intensity $I$,

$$
g_{2}\left(t, t_{0}\right) \equiv \frac{\left\langle I\left(t_{0}\right) I\left(t_{0}+t\right)\right\rangle}{\left\langle I\left(t_{0}\right)\right\rangle\left\langle I\left(t_{0}+t\right)\right\rangle},
$$

and the ensemble-averaged spatial correlation function

$$
C(\delta \mathbf{r}) \equiv \frac{\langle I(\mathbf{r}) I(\mathbf{r}+\delta \mathbf{r})\rangle}{\langle I(\mathbf{r})\rangle^{2}}
$$

where $t_{0}$ is the time when the reference intensity is measured, $\left.t \geq t_{0}\right)$ is the delay time, $\mathbf{r}$ is the coordinate of a pixel on the detector, and $\mathbf{r}+\delta \mathbf{r}$ is the coordinate of another pixel a distance $\delta \mathbf{r}$ away.i $i$ represent the average over the CCD chip. The denominator of the expression

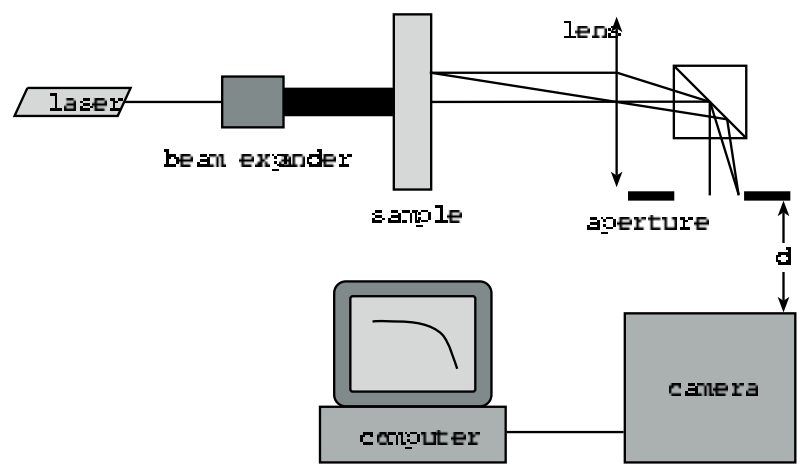

FIG. 1: Experimental set up for the transmission geometry. The laser wavelength is $\lambda=514 \mathrm{~nm}$. We use a Dalsa Camera running at 500 frames/sec and a $500-\mathrm{MHz}$ PC.

on the right hand side of Eq. (11) is the square of the average intensity determined according to the method suggested by Schatzel [16], in order to obtain a slightly more accurate measurement of $g_{2}\left(t, t_{0}\right)$. The algorithms used to calculate these correlation functions are described in greater detail below.

\section{A. Speckle size adjustment}

The size $s$ of the speckles of scattered light formed on the CCD chip is given by

$$
s \simeq d \lambda / a
$$

where $d$ is the distance between the diaphragm and the CCD chip, $\lambda$ is the wavelength of light, and $a$ is the diameter of the diaphragm. By adjusting $d$ and/or $a$, the size of the speckles relative to the size of a pixel on the CCD chip can be controlled. We use a $2 \mathrm{~mm} \times 2 \mathrm{~mm}$ CCD chip with $128 \times 128$ pixels. Each pixel occupies approximately $256 \mu \mathrm{m}^{2}$ with a $100 \%$ fill factor.

To maximize the signal-to-noise, we need to collect data from as many speckles of scattered light as possible. However, if the speckles are too small, for example if several speckles occupy a single pixel, optical contrast is lost and the signal for determining a temporal autocorrelation function $g_{2}(t)$ is degraded. Therefore, it is important to adjust the speckle size so that we obtain a satisfactory optical contrast with as much speckles as possible analyzed.

In order to determine the number of speckles per pixel, we adjust the collection optics (i.e. the size of the iris) and measure the spatial intensity autocorrelation function

$$
C(p)=\frac{\left\langle I_{i} I_{i+p}\right\rangle_{i}}{\left\langle I_{i}^{2}\right\rangle_{i}}
$$

where $I_{i}$ is the intensity of scattered light at pixel $i$ and $I_{i+p}$ is the intensity of light $p$ pixels away. The correlation function $C(p)$ is determined by averaging over all 


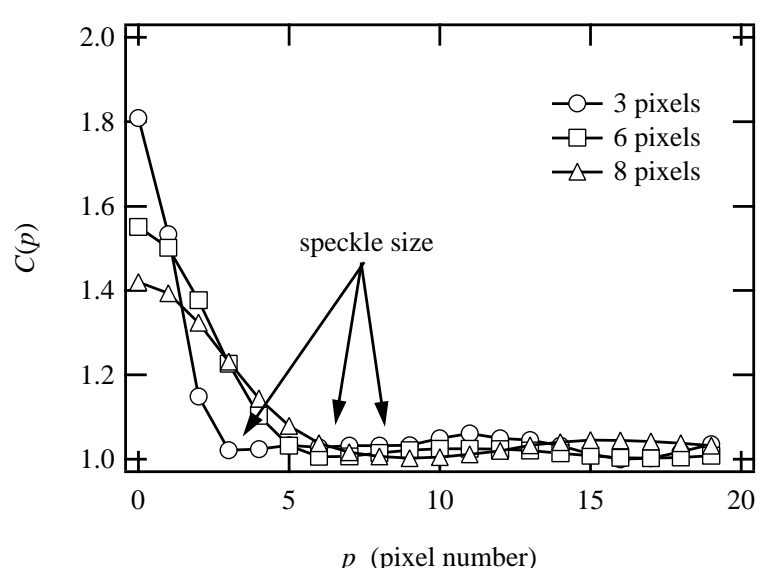

FIG. 2: Spatial correlation function $C(p)$ obtained using different speckle sizes. The first minimum of this function is taken as the speckle spot diameter.

pixels. Figure 2 shows $C(p)$ for three different sets of light collections optics. Note that $C(p)$ first decreases and then oscillates about a baseline intensity. We arbitrarily define the position of the first minimum to be the average speckle size. Hence the speckle size is defined as the distance over which the intensity profile remains spatially correlated.

To determine optimal configuration of the collection optics, we use the static scattering pattern from an immobile glass frit. The frit is sufficiently opaque that diffusion approximation for light transport through the medium is valid. The intercept for the intensity correlation function (i.e. $g_{2}(0)$ ) is expected to lie between 1 and 2. In order to quantitatively evaluate its value, a series of 2000 intercepts was measured. Measurements were performed by self-correlating 2000 images from the glass frit. For each image the mean intensity was calculated as well as the mean squared intensity. The ratio of theses two quantities corresponds to $g_{2}(0)$ for this image. Six series of such measurements were made, each for a different speckle size. Figure 3(a) shows the six distributions of $g_{2}(0)$. The average value $\left\langle g_{2}(0)\right\rangle$ for each speckle size is an evaluation of the contrast, whereas the spread $\Delta g_{2}(0)$ indicates how quickly (i.e. how many images must be correlated before) $g_{2}(0)$ converges to its proper statistical value. As such, $\Delta g_{2}(0)$ quantifies the statistical and instrumental noise. To determine the best compromise between obtaining the highest signal with the lease noise, we plot $\left\langle g_{2}(0)-1\right\rangle / \Delta g(0)$ as a function of speckle size. Figure $3(\mathrm{~b})$ shows that the best configuration is obtained for a speckle size of 3 pixels in diameter. All subsequent measurements are performed in this configuration. The average intercept $\left\langle g_{2}(0)\right\rangle$ allows the determination of the normalization factor given for the Siegert relation:

$$
g_{2}(t)-1=\beta\left|g_{1}(t)\right|^{2}
$$

where $g_{1}(t)=\left\langle E\left(t_{0}\right) E\left(t_{0}+t\right)\right\rangle /\left\langle\left|E\left(t_{0}\right)\right|^{2}\right\rangle$ is the nor-
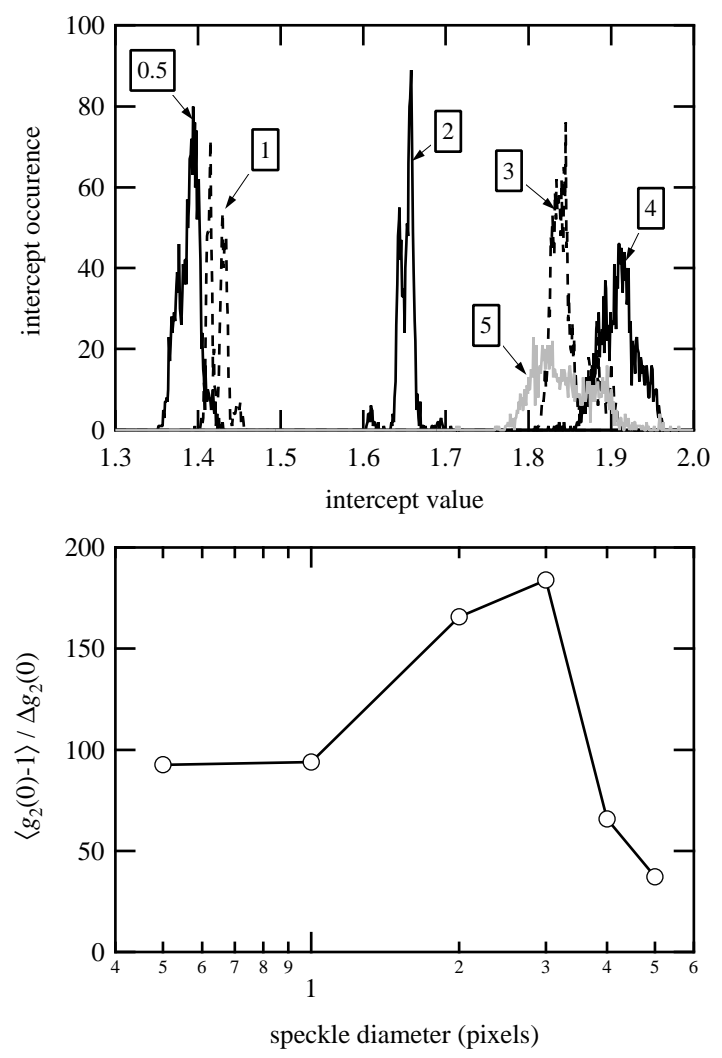

FIG. 3: (a) Histogram of the intercepts $\left(g_{2}(t)=0\right)$ for 2000 correlation functions for a frit at different speckle size. The theoretical intercept should be 2. (b) "Signal-to-noise" $\left\langle g_{2}(0)-1\right\rangle / \Delta g(0)$ as a function of speckle size where $\left\langle g_{2}(0)\right\rangle$ is the average value of $g_{2}(0)$ and $\Delta g(0)$ its spread. It shows that the best compromise between lost of statistics and lost of contrast is obtained for a speckle diameter of 3 pixels.

malized electric field autocorrelation function. We take $\beta=0.82 \pm 0.05$ in subsequent measurements and display our results in terms of the squared electric field correlation function $\left|g_{1}(t)\right|^{2}$ using the Siegert relation above.

\section{CALCULATION ALGORITHM}

In this section we explain how the bitmapped image is processed to calculate the autocorrelation function.

\section{A. Correlation Function Calculation}

At time $t$, a single CCD frame of scattered light is saved in the computer memory (RAM). We call $I_{i}^{\text {raw }}(t)$ the raw intensity of the light incident on pixel $i$ for the image taken at time $t$, and subtract off an average background $\left\langle I_{i}^{\text {back }}\right\rangle$ for pixel $i$ due to stray light and dark counts:

$$
I_{i}(t)=I_{i}^{\text {raw }}(t)-\left\langle I_{i}^{\text {back }}\right\rangle .
$$




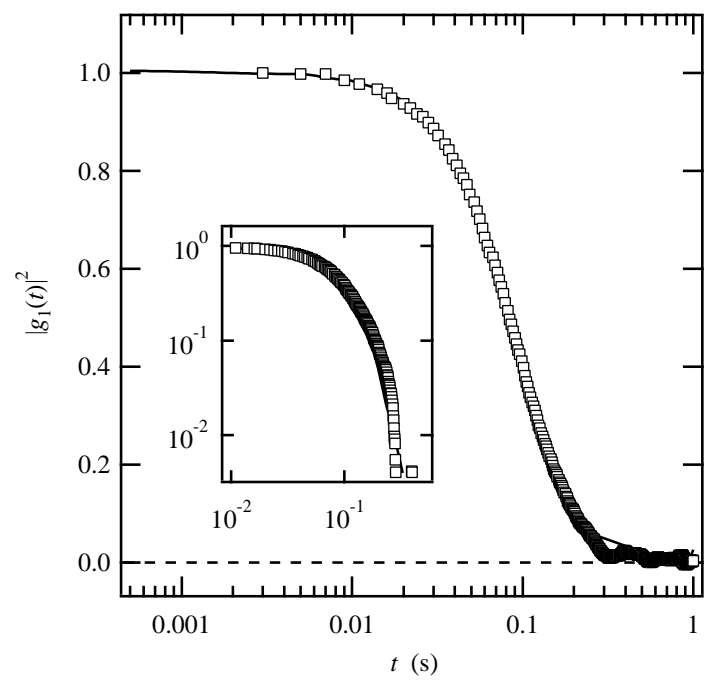

FIG. 4: Comparison of $\left|g_{1}(t)\right|^{2}$ obtained from a sample of $0.5 \mu \mathrm{m}$-diameter beads in pure glycerol for standard singlecorrelator DWS set up (-) and multi-speckle camera based set up (Square). The inert shows the correlation function with the baseline subtracted demonstrating that reliable data is obtained down to $10^{-2}$.

We measure $\left\langle I_{i}^{\text {back }}\right\rangle$ by averaging 1000 images for each pixel with the laser turned off. Further refinements taking into account other sources of extraneous signal can be found in 13. While such refinements prove useful for DLS in the single scattering limit, we found that such corrections to be insignificant for DWS.

One practical problem encountered in acquiring data is that some images may be skipped by the acquisition card due to synchronization problems. Thus, as a precaution, we always record the time $t$ at which each image is obtained.

Applying Eq. (6) to our data, we calculate the correlation function:

$$
g_{2}^{(m)}\left(t_{0}, t\right)=\frac{\left\langle I_{i}\left(t_{0}\right) I_{i}\left(t+t_{0}\right)\right\rangle_{i}}{\left\langle I_{i}\left(t_{0}\right)\right\rangle_{i}\left\langle I_{i}\left(t+t_{0}\right)\right\rangle_{i}}
$$

Note that the average is performed over pixels rather than using the customary method of averaging over the initial time $t_{0}$. That is, the correlation function $g_{2}^{(m)}\left(t_{0}, t\right)$ is calculated by multiplying two frames (one at time $t_{0}$, the other at $\left.t_{0}+t\right)$ together pixel by pixel, summing the results from all pixels, and then dividing by the number of pixels. In general, one frame suffices to obtain a good average for $g_{2}^{(m)}\left(t_{0}, t\right)$ because it consists of a large number of pixels, $16384=128^{2}$ in this case, which constitutes several thousand independent samples from which to determine $g_{2}^{(m)}\left(t_{0}, t\right)$.

The correlation function $g_{2}^{(m)}\left(t_{0}, t\right)$ is obtained in this manner is obtained from an ensemble average of the sample, with no need for the time averaging method typically used with conventional light detection. When data is taken from an ergodic system, both methods give the same result, as illustrated in Fig. A 1 where we compare correlation functions obtained from a CCD camera to one taken with a conventional single detector-single correlator for a sample of polystyrene beads in glycerol obtained undergoing Brownian motion. Significant advantages accrue for the CCD technique, however, when measurements are performed on a non-ergodic sample. Moreover, the CCD method can provide important advantages when measuring correlation functions for nonergodic systems.

\section{B. Multiple correlation function calculation}

In the preceding section, we discussed how a two-time correlation function $g_{2}\left(t_{0}, t\right)$ can be obtained, $t_{0}$ being the time at which the reference image is acquired and $t_{0}+t$ being a later time at which another image is collected and correlated with the reference. Such a correlation function provides a snapshot of the dynamical state of the system at a particular starting time, namely $t_{0}$, and is possible only because sufficient ensemble averaging is achieved by correlating thousands of pixels between two frames from a CCD camera. The ability to obtain such snapshots is particularly useful for studying systems whose dynamics are time-dependent, i.e. non-stationary systems. Examples include systems which are gelling, coarsening, aging, or undergoing some other non-equilibrium non-stationary process. By measuring $g_{2}\left(t_{0}, t\right)$ as a function of the starting time $t_{0}$, the evolving dynamics of such systems can be followed and studied.

Here, we explain a scheme for following the evolving dynamics of a system by measuring many different correlation functions in parallel, each with a different starting time $t_{0}$. A unique and important feature of this scheme is that new correlation functions can be started at intervals as short as $1 / 500 \mathrm{~s}$, while still collecting frames for correlation functions started at earlier times. In fact, up to 500 correlation functions can be collected simultaneously, each with a different starting time $t_{0}$, and without any requirement that a previously started correlation function be finished before starting a new one.

The key to the success of the scheme is the realization that in most cases, it is not necessary to measure the correlation function with the same temporal resolution for all delay times. Typically, small time steps are required for short delay times while larger time steps are required for longer delay times. The scheme we employ takes advantage of this fact. It is essentially an interlacing scheme in which successive frames are sometimes used in different correlation functions, as will be explained below. In effect, the scheme represents a compromise between making maximal use of the information available in the measurements for calculating the correlation functions and obtaining and processing the data from the CCD camera frames at a rapid rate. 
This compromise is necessary because calculating the correlation function between two frames is time consuming; only one pair of frames can be processed (multiplied and averaged) between the acquisition of two successive images. Image stacking is not practical because it quickly leads to memory overflow. Therefore, we employ an algorithm for calculating many correlation functions, labeled by $j=1,2,3, \ldots, j_{\max }$, simultaneously in real time, that makes only the following manageable demands on the computer:

- only one frame multiplication is performed between the acquisition of successive frames,

- storage of a maximum of $j_{\max } \lesssim 500$ reference image frames plus the most recently acquired frame in memory, and

- storage of a maximum of $j_{\max }$ correlation functions in memory, each with on the order of 100 channels.

For a frame rate of 500 frames/s from a $128 \times 128$ pixel CCD camera, these demands can be met by a computer with a Pentium processor running at $500 \mathrm{MHz}$ and possessing 512 MB of RAM. An important feature of the algorithm is that it requires that only the reference frame for each correlation function, along with most recently acquired frame, be stored in the computer at any moment in time; this keeps the number of frames that must be stored in memory to a manageable number. This also allows all correlation functions to be calculated in real time as the experiment proceeds.

The scheme we use is illustrated graphically in Fig. 5. The vertical axis represents the different correlation functions labeled by $j=1,2,3, \ldots, j_{\max }$. The horizontal axis represents the successive frames that are acquired, labeled $n=1,2,3, \ldots$ with each frame being nominally separated in time by the reciprocal $1 / f$ of the frame rate. The reference image for the $j^{t h}$ correlation function is given by image number

$$
n=n_{1}^{(j)}=1+(N+1)(j-1) .
$$

where the subscript " 1 " indicates that this is the first frame of the $j^{\text {th }}$ correlation function. Each of these reference images is stored in memory; $N$ is typically of order 10. The reference image and the next $N-1$ images in sequence are correlated with the reference image. The remaining frames used to calculate the $j^{\text {th }}$ consists of a sequence of selected images as illustrated in Fig. 5. The sequence numbers of the frames used to calculate the $j^{\text {th }}$ correlation function is summarized by the formula:

$$
n=n_{i}^{(j)}= \begin{cases}n_{1}^{(j)}+i-1=(N+1)(j-1)+i & \text { if } 1 \leq i \leq N \\ (j-1)+\frac{1}{2} i(i-1)-\frac{1}{2} N(N-1) & \text { if } i>N\end{cases}
$$

Note that the spacing between the points in a correlation function is constant over the first $N$ points but increases quadratically at later times. The time interval between two successive correlation functions $j$ and $j+1$ is approximately $N / f$ where $f$ is the frame rate. This time interval can be tuned in order to best accommodate the rate of evolution of the system under study. For $N=1$ this time interval can be as short as the inverse frame rate.

In Fig. 6 we show a series of correlation functions obtained using the multispeckle CCD camera technique described above. The correlation functions were obtained from a sample consisting of 0.5 wt.\% alumina $\left(\mathrm{Al}_{2} \mathrm{O}_{3}\right)$ beads, approximately $160 \mathrm{~nm}$ in diameter (AKP30 Sumitomo), that were embedded in a crosslinked elastic network of polybutylacrylate. The first correlation function was started at a time $t_{0}=0$ immediately after a step strain of $5 \%$ was applied to the sample. Thus, the correlation functions measure the subsequent random motion of the beads that arises as a response to the stress within the sample. As the stress relaxes over the course of tens of minutes, the random motion of the beads becomes slower. This slowing down is reflected in the increased decorrelation time of correlation functions started at increasingly later times after the initial step strain. In Fig. 6 we plot the decorrelation time $\tau_{1 / 2}$, defined as the time it takes a correlation function to decay to half its initial value, vs. time, referenced to the starting time $t_{0}$. It exhibits a power law behavior over two decades. A detailed discussion of this experiment will be published elsewhere.

\section{FAST AND SLOW TIME SCALES}

The shortest decorrelation time $\tau_{1 / 2}$ measured in the experiments described in the previous section is fairly slow, approximately $0.1 \mathrm{~s}$. In this particular instance, the frame rate of the camera is sufficient to capture all the dynamics of the process in the correlation function. This is not always the case, however. Many materials, including polymer networks, colloidal gels, and glassy materials possess two characteristic ranges of relaxation times, where only the slower of the two is sufficiently slow for the multispeckle CCD camera method to work. In these cases, the faster relaxation processes are not measured.

To illustrate such a process, we use the multispeckle 


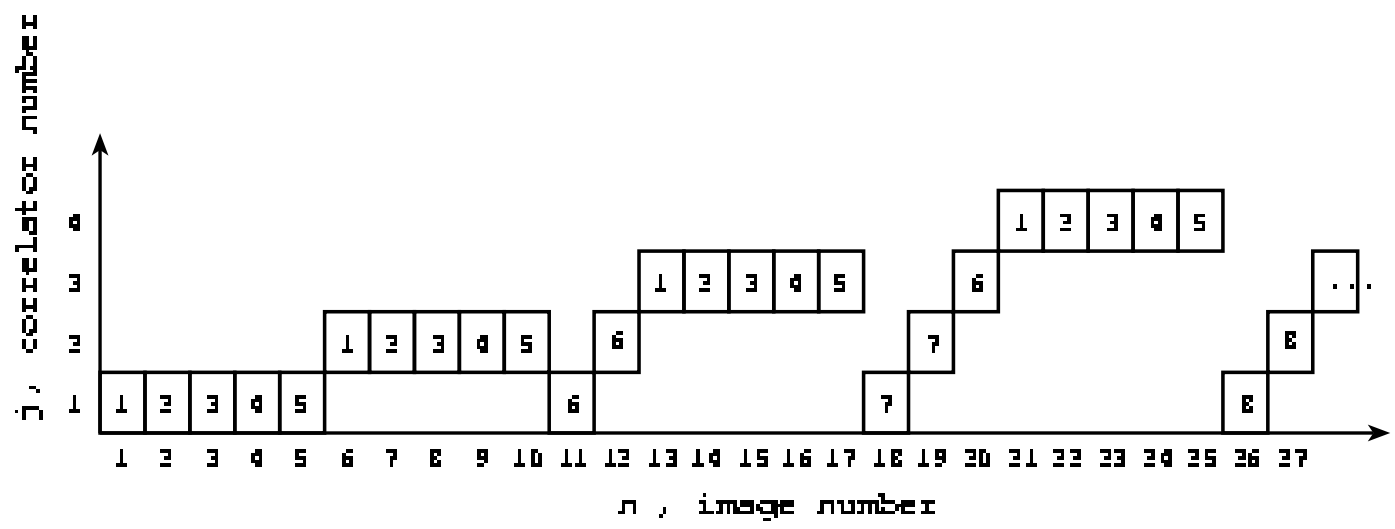

FIG. 5: Schematic representation of image selection and use in the multicorrelator scheme. For this illustration $N=5$. The horizontal axis is the image number $n$ (or $n_{i}^{(j)}$ ) and the vertical axis is the correlator number $j$. The numbers inside the boxes indicate the $i^{\text {th }}$ image used in correlator $j$.

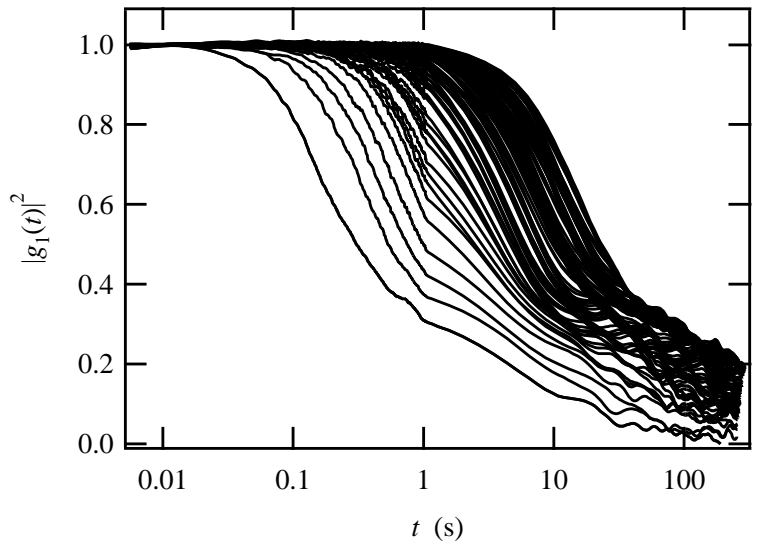

FIG. 6: Correlation functions $\left|g_{1}\left(t_{0}, t\right)\right|^{2}$ taken after a shear step of $5 \%$. Multiple correlation functions are obtained in real time with the ones started later having larger decorrelation times; the decorrelation time increases as the relaxation process slows down. The delay between two successive correlation function is approximately 1 sec.

technique to follow the gelation that occurs following a quench from $60^{\circ} \mathrm{C}$ to $20^{\circ} \mathrm{C}$ in a $1 \%$ gelatin solution containing $1 \%$ latex spheres with a radius of $85 \mathrm{~nm}$. The correlation functions are shown in Fig. 8. As the gelatin gels, the motion of the strands of gelatin and the motion of the spheres embedded within it becomes increasingly restricted. As the gelation proceeds, the height of the plateau and the $y$-intercept of the correlation functions increases. This occurs because there is a fast relaxation process that occurs outside of the time window of the camera. That is, there is an initial rapid drop of $g_{2}(t)$ that occurs on a time scale that is faster than the inverse frame rate of the camera $(\sim 2 \mathrm{~ms})$. Below, we explain how this obstacle can be overcome such that the lower bound of the time window is reduced from $10^{-2}$ to $10^{-8} \mathrm{~s}$.

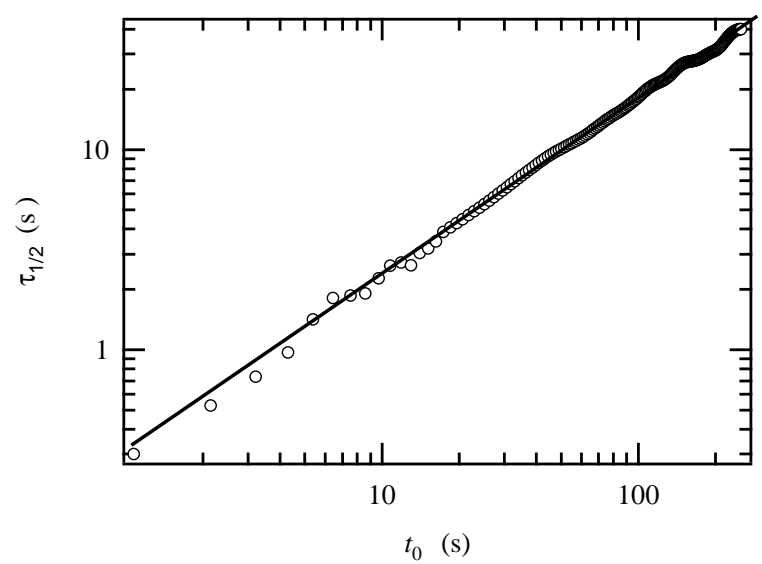

FIG. 7: The correlation time $\tau_{1 / 2}$ vs. the time $t_{0}$, where $\tau_{1 / 2}$ is defined as the delay time where $g_{1}\left(t_{0}, \tau_{1 / 2}\right)=\frac{1}{2}$, and $t_{0}$ is the amount of time that passed after the step strain before starting the correlation function. A new correlation function was started every $1 \mathrm{~s}$. The data are well-described by a power law $\tau_{1 / 2} \sim t_{0}^{0.88}$.

What we would like to be able to do is to simultaneously capture the fast and slow dynamics for a timedependent non-stationary process. Below, we show that this can be accomplished, provided at strict set of criteria are met. Fortunately, it turns out that these criteria are met by most of the systems of interest so that the criteria are not very restrictive in practical situations. As we will show below, we can use a classical photomultiplier-based (fast) correlator, which spans a delay-time range of $10^{-8} \mathrm{~s}$ to about $1 \mathrm{~s}$, to capture the fast dynamics and the multispeckle (slow) correlator to capture the slow dynamics. The key is to properly ensemble average the scattered light signal entering the photomultiplier used with the fast correlator without disturbing the multispeckle signal that enters the slow correlator. 


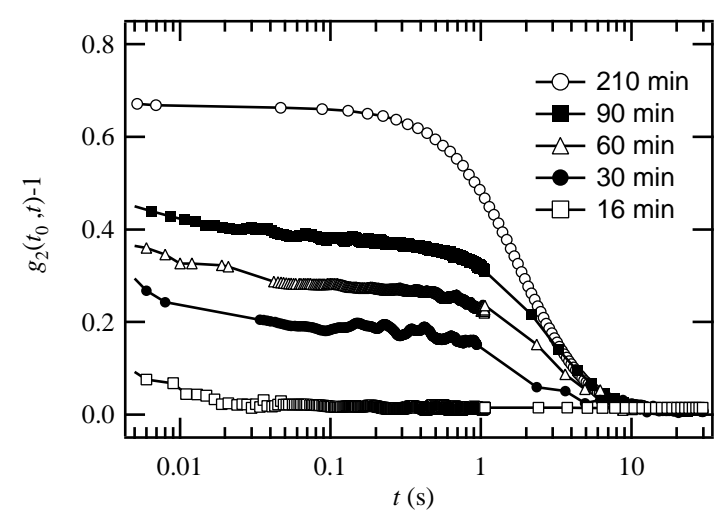

FIG. 8: Correlation functions $g_{2}\left(t_{0}, t\right)-1\left(=\beta\left|g_{1}\left(t_{0}, t\right)\right|^{2}\right)$ started at different times $t_{0}$ (indicated in plot) after the quench of a $1 \%$ gelatine gel with $1 \%$ latex spheres from $60^{\circ} \mathrm{C}$ to $20^{\circ} \mathrm{C}$. The typical evolution time is around 10 minutes. The rise of the intercept and plateau corresponds to the disappearance of the fast liquid phase relaxation process and concurrent increase of the gel elastic modulus.

To provide an ensemble-averaged signal to the fast correlator, we exploit a technique recently developed by Scheffold et al. [8], which enables simple and efficient spatial averaging without requiring any motion of the sample or the detector. This technique is known as the two-cell technique and has been described both theoretically [7] and experimentally [8].

\section{A. Two-cell technique}

The idea of the two-cell technique is to optically couple the studied sample to an ergodic reference system. The light is successively scattered by the sample cell and then by the reference cell before entering the photomultiplier. This can be implemented simply by illuminating two cells in series, and detecting the light that passes through and is scattered by both samples in the two cells. In that case, the non-ergodic signal emerging from the sample cell is made ergodic by its scattering through the reference cell. The second cell can thus be regarded as performing a slow spatial averaging of the sample signal. Under carefully specified optical conditions, the correlation function, $g_{1}^{M}(t)$, measured after passing through the two cells is simply the product of the correlation function of each cell:

$$
g_{1}^{M}(t)=g_{1}^{S}(t) \times g_{1}^{R}(t),
$$

where the superscripts $R$ and $S$ stand for the sample and the reference correlation functions, respectively. The conditions under which the two-cell technique can be employed have been carefully considered in Ref. [7]. They find that the primary condition required is that each photon traverses the space between the sample and reference cells only once. This means that for a configuration where a significative amount of light is lost between the two cells, as was done in Ref. [8], this condition is satisfied when $l_{R}^{*} / L_{R} \gg l_{S}^{*} / L_{S}$, where $l_{i}^{*}$ and $L_{i}$ are, respectively, the photon transport mean free path and the cell thickness for the reference $(i=R)$ and the sample $(i=S)$ cells.

\section{B. Experimental set up}

Figure 9 illustrates how we incorporate the two-cell technique into the multispeckle correlator so that we can simultaneously capture the fast and slow dynamics from a slowly evolving non-ergodic system. We arrange the optics for the multispeckle (slow) correlator just as before so that surface from which the multiply scattered light exits the sample cell is imaged with a 1:1 ratio onto a pinhole behind which the CCD camera is placed. For the fast correlator, a non-polarizing beam splitter is placed a short distance in front of the pinhole so that half of the light is diverted onto the reference cell. The reference cell positioned so that the exit plane of the sample cell is imaged onto the reference cell's front surface. An single mode optical fiber with a collimator collects the scattered light exiting the reference cell at an angle of approximately $15^{\circ}$ and sends it to a photomultiplier whose output is connected to a multiple-sample-time correlator.

The reference cell has a thickness of $5 \mathrm{~mm}$ and contains a glycerol solution of $0.5 \mu$ m-radius latex spheres with a volume fraction of $1 \%$. The concentration of spheres is chosen so that there is just enough multiple scattering in the reference cell to ensure that essentially no light passes through the sample without being scattered at least several times but with $l_{R}^{*} / L_{R}$ as big as possible. However, this must be done so that the decorrelation time of the reference correlation function is somewhat larger, typically by a factor of about 3 to 10 , than the inverse frame rate of the CCD camera. By adjusting the concentration of spheres and the viscosity of the solution with glycerol, these conditions are readily achieved.

Before a measurement can be made using the two-cell technique, it is necessary to measure the correlation function of the reference cell in the absence of the sample cell and to ensure that its correlation time is greater than the inverse frame rate. Data from such a measurement is shown in Fig. 10, from which one can see that the decay time of $g_{1}^{R}(t)$ is about $0.1 \mathrm{~s}$. The correlation function $g_{1}^{M}(t)$ is then measured with the sample cell in place, from which the sample correlation function $g_{1}^{S}(t)$ is obtained using Eq. (10). Figure 10 shows results from a typical set of measurements, including the reference cell correlation function $g_{1}^{R}(t)$, the measured two-cell correlation function $g_{1}^{M}(t)$, and the true correlation function of the sample $g_{1}^{S}(t)$ obtained from Eq. (10). Note that $g_{1}^{S}(t)$ is obtained accurately from the first time measured by the correlator, about $10^{-7} \mathrm{~s}$, out to about $30 \mathrm{~ms}$, or approximately the decorrelation time of $g_{1}^{R}(t)$. For longer 


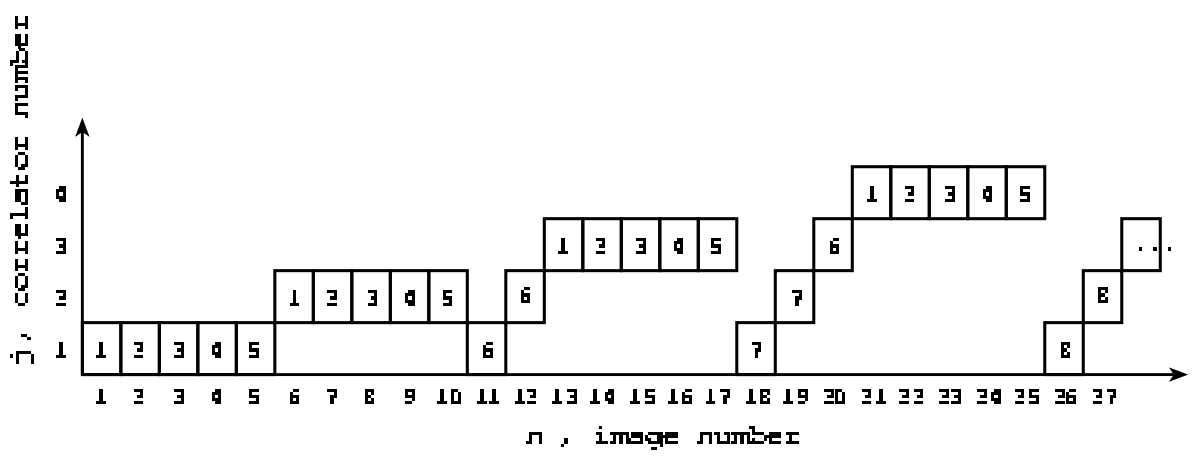

FIG. 9: Schematic for the two-cell technique. A single mode optic fiber and a multi-delay-time correlator with a photomultiplier tube are used. The second cell is made with $0.5 \mu \mathrm{m}$ polystyrene spheres in a dilute solution of glycerol. The polarizing cube has been replaced by a non-polarizing beam splitter.

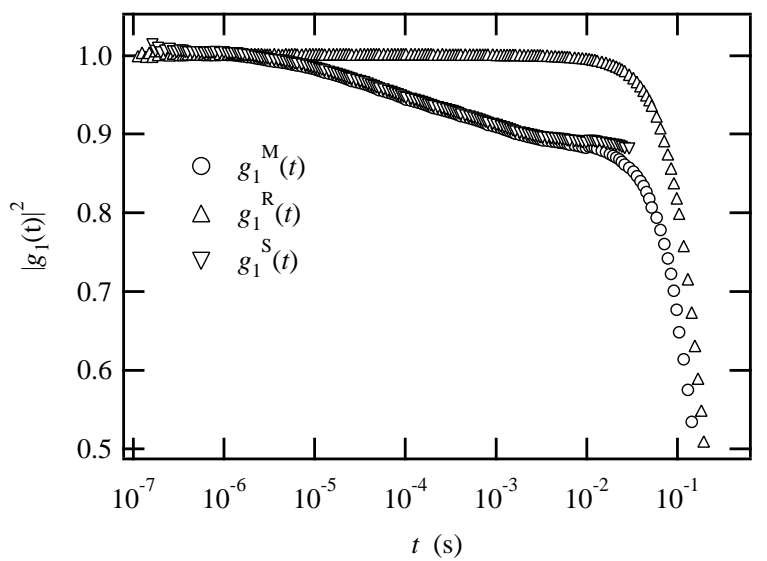

FIG. 10: Correlation functions $\left|g_{1}^{M}(t)\right|^{2}[\circ],\left|g_{1}^{R}(t)\right|^{2}[\triangle]$ $\left|g_{1}^{S}(t)\right|^{2}[\nabla]$ for the gelatine gel after 210 min. The sample correlation function $\left|g_{1}^{S}(t)\right|^{2}$ displays first a rapid decay and then a plateau due to the trapping of the beads in the gel network.

delay times, the reference correlation function $g_{1}^{R}(t)$ falls to zero making recovery of $g_{1}^{S}(t)$ using Eq. (10) impossible. Note that the sample correlation function $g_{1}^{S}(t)$ determined in this way has a significant time interval of overlap, from about $2 \mathrm{~ms}$ to about $30 \mathrm{~ms}$, with the correlation function measured by the multispeckle technique. Thus, it should be possible to superimpose the correlation functions from the two techniques to obtain a single correlation function covering the entire range of time scales from $10 \mathrm{~ns}$ to hundreds of seconds or longer. It is important to note, however, that in order from the signal to be ergodic the correlation function obtained from the twocell technique to be properly ensemble averaged, data must be collected over a time that is about $10^{3}$ times larger than the decay time of the reference correlation function. In our case the acquisition time is $100 \mathrm{~s}$.

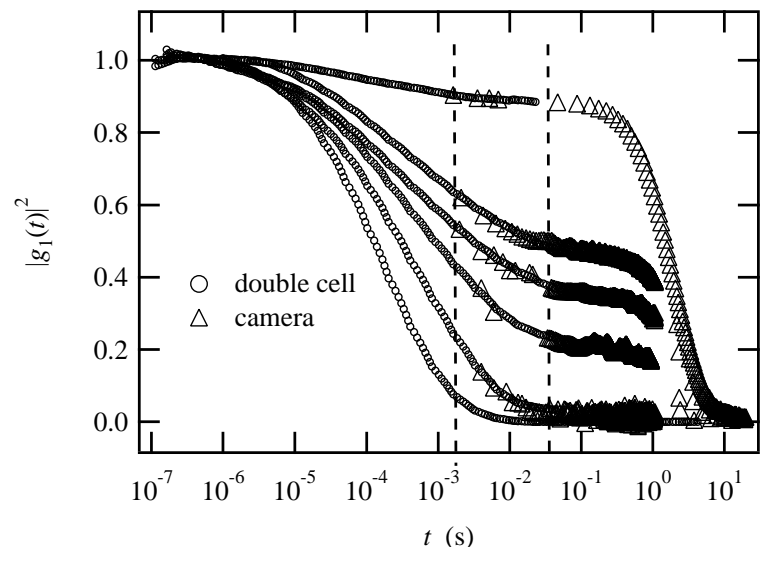

FIG. 11: Normalized correlation functions obtained using the double cell $(\circ)$ and multispeckle $(\triangle)$ techniques. The range of overlap is indicated by the dashed lines.

\section{Overlapping data from the two techniques}

In order to overlay the correlation functions measured by the two techniques, they must be properly normalized using the respective Siegert coefficients $\beta$ for each measurement (see Eq. 5). For the multispeckle CCD camera method, $\beta=0.8$, as discussed in Section IIIA. For the double cell technique, $\beta$ is determined by the value of the intercept from the correlation function of the reference cell alone. For the reference cell, we obtain $\beta=0.95$.

Figure 11 shows correlation functions from the two techniques for the gelation experiment described in Section $\mathrm{V}$. The acquisition time for each double cell measurement was $100 \mathrm{~s}$. Excellent agreement is obtained over the range where the two techniques overlap $(2 \mathrm{~ms}-$ $30 \mathrm{~ms}$ ). Note that the overlap occurs in a region where the slope of the correlation function is sizeable, making the agreement even more impressive.

Finally, it us useful to compare the results for different methods of measuring a correlation function. In Fig. 


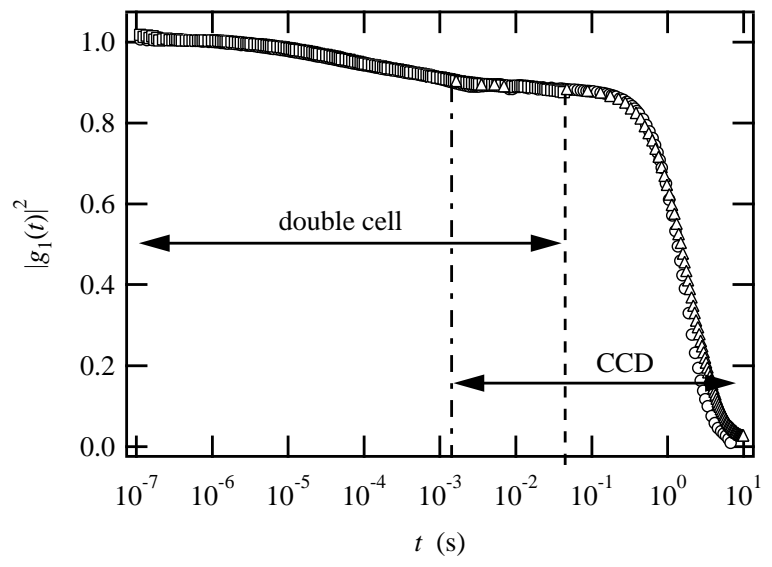

FIG. 12: Comparison of correlation functions obtained using a single correlator without a reference cell (conventional single-cell method - $3000 \mathrm{~s}$ ) [0], with a reference cell (two-cell method - $100 \mathrm{~s}$ ) [Square], and using multispeckle CCD camera $(10 \mathrm{~s})[\triangle]$ techniques. Discrepancies at long time at are attributed to poor statistics for the the conventional method.

12, we plot three correlation functions: (1) one obtained using the multispeckle technique with a window of decay times of $2 \mathrm{~ms}-10 \mathrm{~s}$. Averaging was performed in real time (up to the longest decay time or $\sim 20 \mathrm{~s}$ ); (2) another obtained using the double cell technique over the decay-time window of $10^{-7} \mathrm{~s}-30 \mathrm{~ms}$ and averaged for $100 \mathrm{~s}$; (3) and a third obtained using a classical set-up with a photomultiplier tube and single correlator over the decay-time window of $10^{-7}-5 \mathrm{~s}$ and averaged for $3000 \mathrm{~s}$. The measurements using the double cell and the multispeckle methods were done simultaneously; the measurement with the photomultiplier and single correlator was performed just after the other two, simply by removing the reference cell. The agreement is very good over the time window of $10^{-7}-10^{-1} \mathrm{~s}$. There are deviations, however, between the single correlator and the multispeckle techniques at longer delay times. These deviations occur because data obtained using the single correlator technique must be collected over a fairly long data-acquisition time, $100 \mathrm{~s}$ in this case, in order to obtain satisfactory signal-to-noise. In cases where the dynamics of the sample evolves with time, the multispeckle technique has clear advantages.

The multi-speckle technique can measure correlation functions in real time for delay-times larger than $2 \mathrm{~ms}$. When coupled simultaneously (in the same set-up) with the two-cell technique, the combined method can measure correlation functions over an extremely broad range of delay times. As a demonstration, we show in Fig. 13 a correlation function measured for a gel of silica beads ( $0.2 \mu \mathrm{m}$ in diameter) at a volume fraction of $52 \%$ with an Ionic force adjusted to $1.410^{-} 2 \mathrm{M}$ with $\mathrm{KNO}_{3}$. correlation function spans a range of delay times from $10^{-8} s$ to greater than $10^{3} \mathrm{~s}$, or more than 11 decades in time. Such a measurement is not only remarkable, it is extremely useful for probing glassy systems which can exhibit dy-

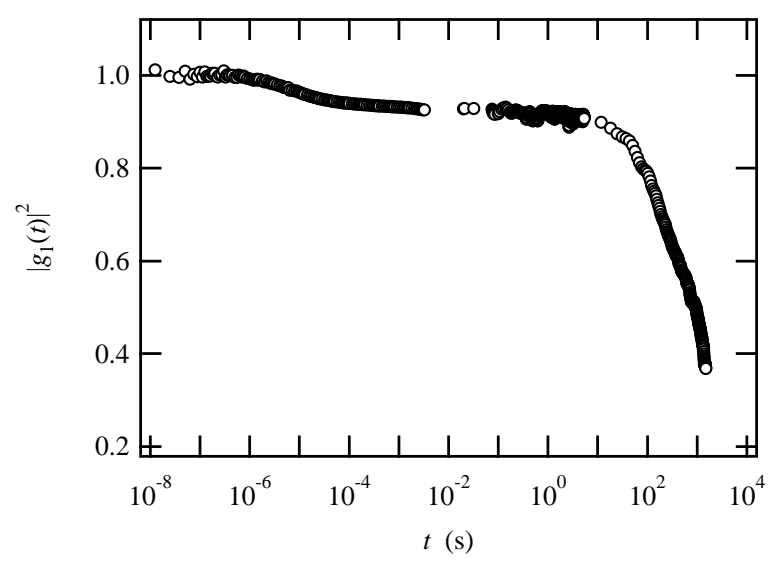

FIG. 13: Correlation function $\left|g_{1}(t)\right|^{2}$ for a suspension of silica spheres $(\phi=22 \%)$. The fast part of the correlation function was measured using the two-cell technique and the slow part using the multispeckle technique. The combined measurements span 11 decades.

namics over a vary broad range of delay times.

Lastly, there are a couple of practical constraints that bear mentioning. First, the two techniques can be usefully coupled only if the typical time scale over which the system evolves is much longer than the averaging time required for the two cell technique. As a practical matter, the required averaging time is set by the longest delay time one wishes to measure. That time is determined primarily by the desire to have some range of delay times where the two-cell and multi-speckle techniques overlap. The limitation here is the speed of the camera that can be used for the multi-speckle technique. Second, the simultaneous use of the two techniques leads to a certain amount of light loss. Thus, a powerful laser $(\sim 1 \mathrm{~W})$ is recommended.
[1] G. Maret and P. E. Wolf. Multiple light scattering from disordered media. the effect of brownian motion of scatterers. Zeitschrift fur Physik B, 65(4):409-413, 1987.

[2] D. J. Pine, D. A. Weitz, P. M. Chaikin, and E. Herbolzheimer. Diffusing-wave spectroscopy. Physical Review Letters, 60(12):1134-1137, 1988.
[3] D.A. Weitz and D.J. Pine. Diffusing-wave spectroscopy. In Wyn Brown, editor, Dynamic light scattering: The method and some applications, volume 49 of Monographs on the physics and chemistry of material, pages 652-720. Oxford University Press, Oxford, 1993.

[4] P. N. Pusey and W. Van Megen. Dynamic light scattering 
by non-ergodic media. Physica A, 157(2):705-741, 1989.

[5] J. G. H. Joosten, E. T. F. Gelade, and P. N. Pusey. Dynamic light scattering by nonergodic media: Brownian particles trapped in polyacrylamide gels. Physical Review A, 42(4):2161-2173, 1990.

[6] J.Z. Xue, D.J. Pine, S.T. Milner, X.-l. Wu, and P.M. Chaikin. Nonergodicity and light scattering from polymer gels. Physical Review A, 46(10):6550-6563, 1992.

[7] S. E. Skipetrov and R. Maynard. Dynamic multiple scattering of light in multilayer turbid media. Physics Letters A, 217(2-3):181-185, 1996.

[8] S. Romer, F. Scheffold, and P. Schurtenberger. Sol-gel transition of concentrated colloidal suspensions. Physical Review Letters, 85(23):4980-4983, 2000.

[9] F. Scheffold, S. E. Skipetrov, S. Romer, and P. Schurtenberger. Diffusing-wave spectroscopy of nonergodic media. Physical Review E, 63(6, pt.1-2):061404/1-11, 2001.

[10] A. P. Y. Wong and P. Wiltzius. Dynamic light scattering with a ccd camera. Review of Scientific Instruments, 64(9):2547-2549, 1993.

[11] E. Bartsch, V. Frenz, J. Baschnagel, W. Schartl, and H. Sillescu. The glass transition dynamics of polymer micronetwork colloids. a mode coupling analysis. Journal of Chemical Physics, 106(9):3743-3756, 1997.

[12] O. K. C. Tsui and S. G. J. Mochrie. Dynamics of concentrated colloidal suspensions probed by x-ray correlation spectroscopy. Physical Review E, 57(2 PTB):2030-2034, 1998.

[13] L. Cipelletti and D. A. Weitz. Ultralow-angle dynamic light scattering with a charge coupled device camera based multispeckle, multitau correlator. Review of Scientific Instruments, 70(8):3214-3221, 1999.

[14] L. Cipelletti, S. Manley, R. C. Ball, and D. A. Weitz. Universal aging features in the restructuring of fractal colloidal gels. Physical Review Letters, 84(10):2275-2278, 2000.

[15] A. Knaebel, M. Bellour, J. P. Munch, V. Viasnoff, F. Lequeux, and J. L. Harden. Aging behavior of laponite clay particle suspensions. Europhysics Letters, 52(1):7379, 2000.

[16] K. Schatzel and E. O. Schulzdubois. Improvements of photon correlation techniques. Infrared Physics, 32:409 416, 1991. 


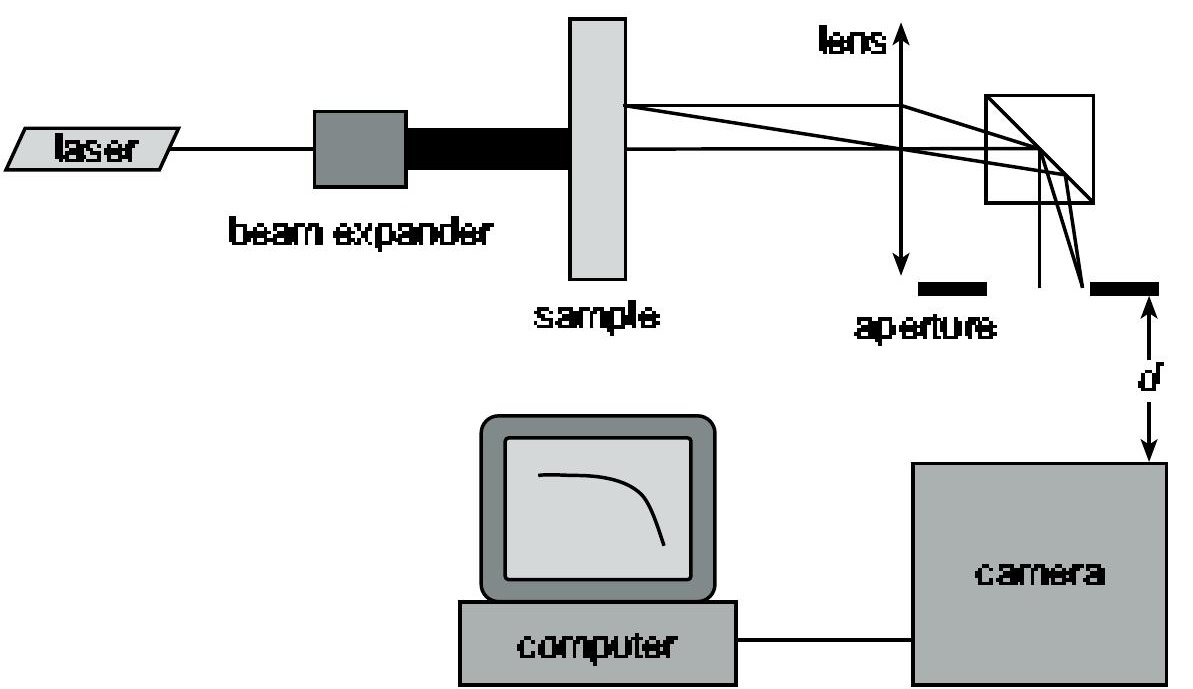

